\title{
Pipelining and Optimal Routing Techniques for Improving the Performance of Wireless Networks
}

\author{
Kumar. V \\ Department of CSE \\ Knowledge Institute of Technology \\ Salem, Tamilnadu, India
}

\author{
Balasubramanie. $\mathrm{P}$ \\ Department of CSE \\ Kongu Engineering College \\ Perundurai, Tamilnadu, India
}

\begin{abstract}
In this thesis, a cross layer network design approach using effective MAC layer and optimal Network layer design has been proposed and implemented to improve the performance of the wireless networks. For this purpose, new MAC design principles have been proposed for effective scheduling and channel allocation where pipelining techniques proposed for improving the channel utilization. In addition, a new bandwidth allocation technique using partitioning has been proposed in this paper which allows varying the amount of traffic dynamically by increasing or decreasing the window size depending on the traffic. Finally, an ant colony based routing algorithm which uses cluster based routing has been proposed and implemented to match the newly designed MAC layer.
\end{abstract}

\section{Keywords}

Pipelining, Optimal Routing, Ant Colony Optimization, Cross layer Design.

\section{INTRODUCTION}

Wireless Networks have become the most important access network technologies internetworking area. Wireless networks can operate in two modes namely infrastructure mode and ad hoc mode. In wireless networks that have infrastructures with base stations, communication typically takes place between wireless nodes and base stations, but not directly between wireless nodes. The presence of base stations simplifies routing and resource management in infrastructure based wireless networks as the routing decisions are made in a centralized manner. On the other hand, a set of protocols are provided to handle routing effectively. Moreover, routing plays an important role in the coordination between the MAC layer and the network layer. Therefore, a cross layer network design that considers channel allocation, packet scheduling and routing is an important design criteria in wireless networks because the performances of many applications are heavily dependent on the efficiency of these protocols. The efficiency of the routing protocol in turn depends on the overhead involved in route discovery and route maintenance [4]. Since the overall performance of the network mainly depends on routing protocols and flow control protocols present in the data link layer, a cross layer design through a MAC layer protocol which can coordinate with the routing protocols can improve the throughput of the network.

This paper proposes an efficient MAC protocol for wireless Mobile networks that provides effective Channel allocation techniques in coordination with the an optimal routing at the network layer for effective cross layer design. Since, wireless networks work on two modes namely with access points and ad hoc networks where access points are not used, this cross layer design provides protocols which will suite both these two modes. In this way, the performance of the network has been improved considerably by the protocols proposed in this work for each layer. This type of cross layer is necessary since, if the performance of one logical layer is improved without considering the neighboring layer, then only a negligible amount of improvement in throughput can be achieved. For example, if MAC layer has a good performance but the network layer doesn't provide a matching performance the overall performance will be affected. In the sender side, the network layer must deliver the packets fast in a reliable manner to the MAC layer so that pipelining can take advantage of sending such speedy arrival of packets in order to improve the overall throughput. Similarly, if the segments coming from MAC layer of the receiver side are not considered for routing, packet delivery ratio will not be improved. Hence, a cross layer design has been carried out in this work in order to improve the overall performance of the network.

In order to implement the cross layer design proposed in this paper, new channel allocation methods have been proposed using partitioning and pipelining that improves the performance of 802.11 MAC. Moreover, an extended ant colony optimization technique is used to improve the route discovery process of AODV. In addition, a cluster based routing algorithm that helps to reduce the number of messages sent in the route discovery process has been proposed and implemented in order to improve the performance of the network.

The remainder of this paper is organized as follows: Section 2 provides a survey of related works in this area. Section 3 depicts the architecture of the system proposed in this paper. Section 4 discusses the cross layer design methodologies used in this work. Section 5 presents the results obtained in this work and compares them with the existing works. Section 6 gives the conclusions on this work and suggests some possible future works.

\section{LITERATURE SURVEY}

There are many works on MAC layer design that are found in the literature [1][2][3]. Sunil Kulkarni and Aravind Iyer [1] proposed an address-light, integrated MAC and routing protocol (abbreviated AIMRP) for wireless sensor networks (WSNs). 
They proposed this protocol for detecting rare events which require prompt detection and response. AIMRP organizes the network into concentric tiers around the $\operatorname{sink}(\mathrm{s})$, and routes event reports by forwarding them from one tier to another, in the direction of (one of) the sink(s). AIMRP is address-light in that it does not employ unique per-node addressing, and integrated since the MAC control packets are also responsible for finding the next-hop node to relay the data, via an any-cast query. For reducing the energy expenditure due to idle-listening, AIMRP provides a power-saving algorithm which requires absolutely no synchronization or information exchange. They evaluated AIMRP through analysis and simulations, and compared it with another MAC protocol proposed for WSNs called S-MAC. However, they have focused only on power optimization in wireless sensor networks.

Michele Rossi and Michele Zorzi [3] presented integrated MAC/routing solutions for wireless sensor networks. In their system, at the MAC layer, every node accesses the channel according to its own cost by means of properly defined costdependent access probabilities. They have used Costs to capture the suitability of a node to act as the relay and may depend on several factors such as residual energies, link conditions, queue state, etc. Their cost-aware MAC discriminates nodes right in the channel access phase and thereby assisting the forwarding decisions to be made at the routing level. Kitae Nahm et al [5] investigated the cross-layer interaction between TCP and routing protocols in the IEEE 802.11 ad hoc networks. They proposed two complementary mechanisms namely, the TCP fractional window increment (FeW) scheme and the Route-failure nOtification using BUlk-losS Trigger (ROBUST) policy to address the cross layer design issues. The TCP FeW scheme is a preventive solution used to reduce the congestion-driven wireless link loss. The ROBUST policy is a corrective solution that enables on-demand routing protocols to suppress overreactions induced by the aggressive TCP behavior. They have shown by computer simulation that these two mechanisms result in a significant improvement of TCP throughput without modifying the basic TCP window or the wireless MAC mechanisms.

Atef Abdrabou and Weihua Zhuang [6] proposed a model-based quality-of service (QoS) routing scheme for IEEE 802.11 ad hoc networks. Unlike most of QoS routing schemes in the literature, their scheme provides stochastic end-to-end delay guarantees, instead of average delay guarantees, to delay-sensitive bursty traffic sources. Via a cross-layer design approach, the scheme selects the routes based on a geographical on-demand ad hoc routing protocol and checks the availability of network resources by using traffic source and link-layer channel modeling, taking into consideration the IEEE 802.11 characteristics and node interactions. Their scheme extends the well developed effective bandwidth theory and its dual effective capacity concept to multihop IEEE 802.11 ad hoc networks. However, this cross layer design can be further enhanced with cluster based routing for providing more effective QoS.

Jinhua Zhu et al [2] proposed a unified MAC and routing framework to exploit the temporal and frequency resources to significantly improve the throughput of ad hoc networks. Their joint channel assignment and routing scheme searches for an efficient transmission path, taking into account the constraints due to the limited number of available channels and radio interfaces and the impact of MAC-layer scheduling. This work is a milestone for cross layer design. However, the routing performance can be enhanced further by using a cluster based routing algorithm.

Correia et al.[7] proposed a Mobility-aware Ant Colony Optimization Routing for Vehicular Ad Hoc Networks Vehicular Ad hoc Networks (VANETs). Their work proposes an Ant Colony Optimization (ACO) procedure that takes advantage of information available in vehicular networks - such as the vehicles' position and speed in order to design an antbased algorithm that performs well in the dynamics of such networks. The results obtained by them suggest that making use of environmental information can make ACO algorithms more suitable for routing in vehicular ad hoc networks.

In this paper, an attempt has been made to improve the performance of MAC Layer by pipelining. Since, pipelining allows the execution of various jobs to overlap in time, the number of packets transmitted is increased. Moreover, pipelining is accomplished by dividing each job into stages and as soon as the first job finishes a stage, the second job enters that stage and the first job moves on to the next stage. This work proposes an ant colony based route discovery mechanism and a cluster based routing algorithm which can coordinate with the MAC layer for providing effective QoS.

Routing in wireless ad hoc networks has a number of challenges. To address the challenge of cross layer design, Chilukuri Shanti et. al [8] presented an integrated MAC and routing protocol called Delay Guaranteed Routing and MAC (DGRAM) for delay-sensitive wireless sensor network (WSN) applications. Laura et al [9] proposed an ant based routing algorithm for routing in mobile ad hoc networks.

However, most of the work has been carried out in the scope of the IEEE 802.11 standards only. However, it is necessary provide substantial work in the area of parallel scheduling and cluster based routing in order to improve the network performance. In this work, a novel framework for effective routing and channel allocation has been proposed. The routing module provides new route discovery and route maintenance techniques using cluster formation and cluster based routing techniques.

\section{SYSTEM ARCHITECTURE}

The architecture of the proposed system given in Figure 1 has two major subsystems called clustering and routing subsystems. The clustering subsystem has three components namely cluster formation module, cluster maintenance module and Ant Colony Optimization module. The cluster formation module creates the cluster structure and the cluster maintenance module updates the cluster structure according to the changes taking place in the topology of the network. Based on the information present in the clustering structure, the routing subsystem discovers the routing information, stores them in the routing table and updates them. The routing module sends the packets to the pipelining module for scheduling and channel allocation. In this way the clustering subsystem and routing subsystem work together to provide fast delivery of packets to the MAC Layer where pipelining is used for effective channel allocation. 


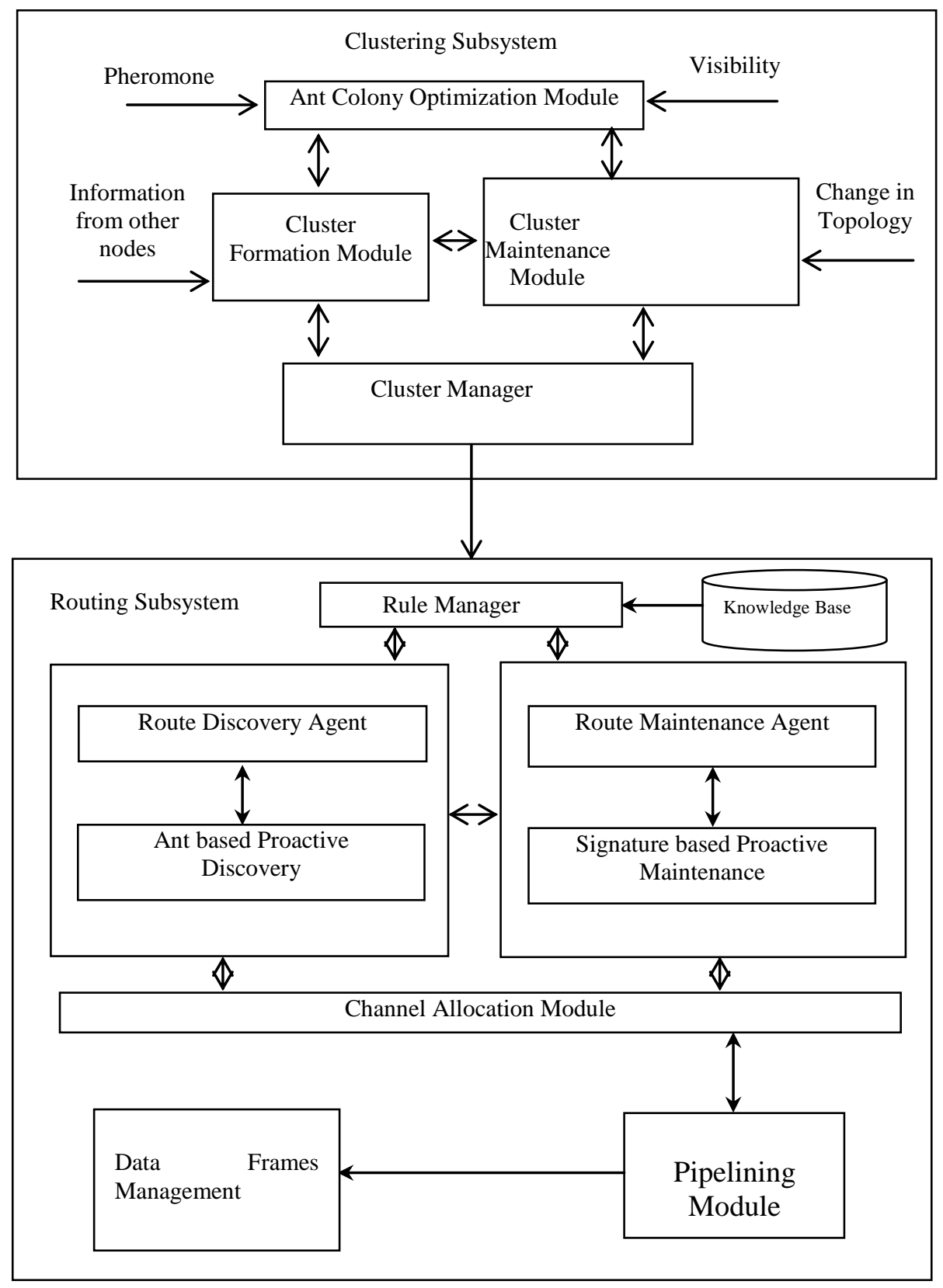

Fig 1: Overview of System Architecture

\section{PROPOSED ALGORITHM}

Two algorithms have been proposed in this paper for effective scheduling of frames in the MAC layer and suitable routing with clusters and ant colony optimization in the network layer.

\subsection{Pipelined Scheduling}

In the new pipelining technique proposed for the MAC layer, the process is divided into two steps namely, contention resolution and packet delivery. In contention resolution, two steps are proposed namely contention resolution stage 1 and stage 2 . When a transmission cycle begins, some stations enter stage 1 and then they enter the stage2. When the first set of stations enters stage2, the next set of all stations come to stage 1 . In this way, multiple stations are allowed to transmit simultaneously using pipelining as shown in Figure 2.

The new technique differs from 802.11 DCF only in the contention resolution procedure. The other functions remain the same. 


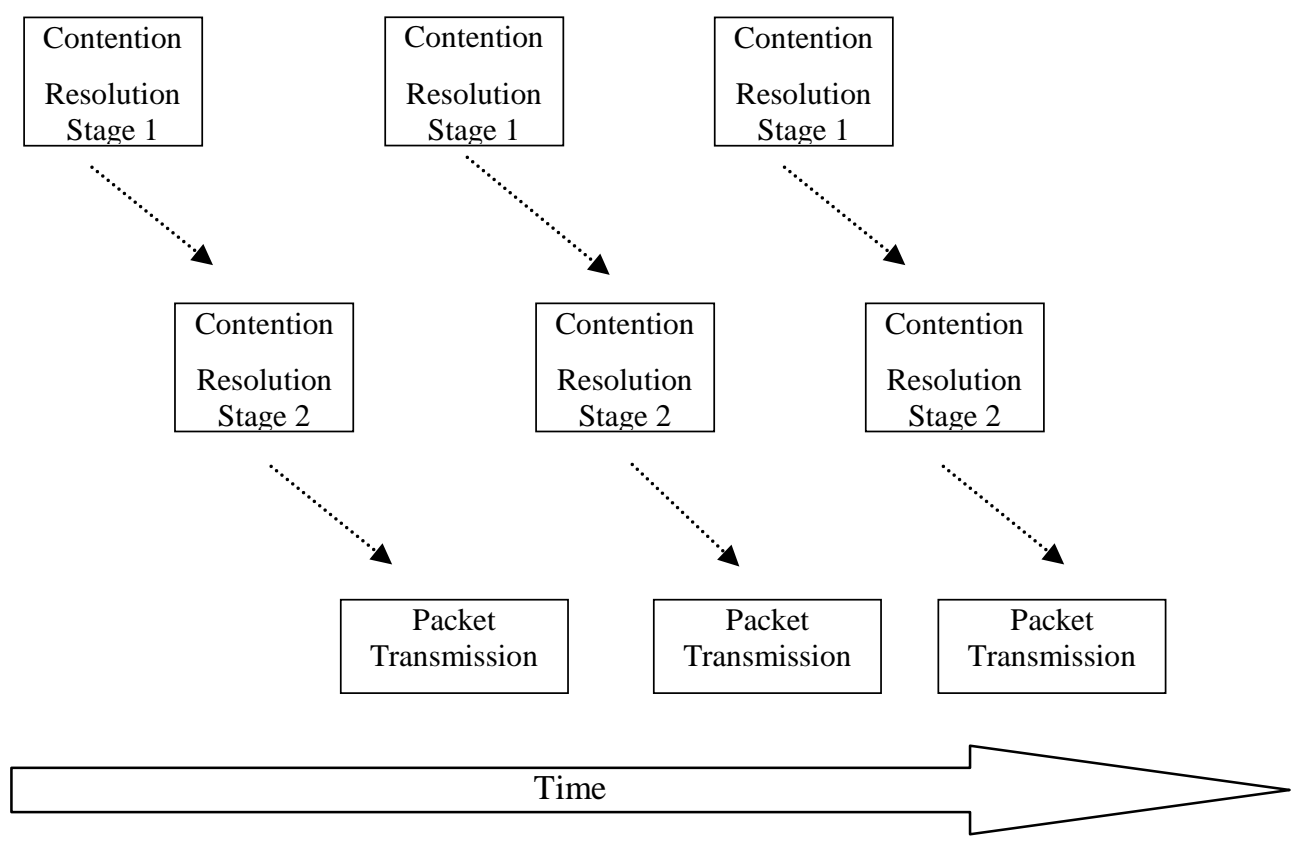

Fig 2: Proposed Pipelining Technique

In contention resolution stage 1, a back-off counter, named bc1, is set which is associated with the first contention resolution stage. bc1 is chosen to be uniformly distributed over the interval [0, CW1]. CW1 is the contention window for stage 1 and has a minimum value $C W 1$ min and maximum value $C W 1$ max. In this work, CW1min is taken as 0 and CW1max is set as 1023 . This parameters were chosen by conducting experiments with 8,16 , $32,64, \ldots ., 1024$ nodes. The initial value of bc1 is $\mathrm{CW} 1 \mathrm{~min}$. This back-off counter bc1 is counted down in two ways:

1. At the beginning of a transmission cycle, each active station reduces bc1 by $\mathrm{K}$, except for the station which just finished transmitting a packet. Since a station will reset CW1 to CW1min after a successful transmission, if it immediately reduces bc 1 by $K$, this station will have more chances than other stations to enter stage 2.

2. During a transmission cycle, each active station reduces its bc1 by 1 after each idle slot.

Whenever a station's bc1 becomes less than or equal to 0 , this station enters into stage 2 . If a station's bc1 is counted down to zero during a transmission cycle (i.e., by using the second method above for decrementing bc1 by 1 ), it can be said that this station "interferes" with the second stage. With a suitable choice of $\mathrm{K}$, interference only happens to a small fraction of stations entering stage 2. Now if there are $M$ stations that enter stage 2 in a transmission cycle, among the $\mathrm{M}$ stations, one station will eventually win channel access. The remaining $M-1$ stations in stage 2 will return to the first stage, double CW1 and regenerate bc1. The winning station will finish its packet transmission, return to the first stage, reset CW1 to CW1min and regenerate bc1. Those stations which do not enter the second stage will not participate in channel contention and hence the CW1 value will not get changed.
A back-off counter, named bc2, is associated with the second contention resolution stage. If a station enters stage 2 during a transmission cycle, it will set the initial value of bc2 to 0 . On the other hand, if a station enters stage 2 at the beginning of a transmission cycle (i.e., bc1 is less than or equal to zero after reducing by $\mathrm{K}$ ), it will generate an initial value for bc 2 which is uniformly distributed over the interval [0, CW2]. CW2 is contention window for the second stage. It has minimum value $\mathrm{CW} 2$ min and maximum value CW2 max. The initial value of CW2 is CW2min. After the channel has been idle for DIFS duration, each station in stage 2 decreases bc 2 by 1 after each slot. When bc2 reaches zero and the channel is idle, the station will begin its transmission. Before the bc 2 of a station reaches zero, if a frame sent by some other station is successfully received or overheard (e.g., RTS or CTS frames in the case of the RTS/CTS access method), the former station will return to stage 1 and double its CW1. When a collision happens, the colliding stations will double their CW2 and generate a new bc2 value from the interval $[0, \mathrm{CW} 2]$. They will remain in the second stage and repeat the above channel contention procedure for stage 2 until someone wins the channel.

In the packet transmission stage, data and acknowledgment transmission takes place. Data are transmitted from source to destination and in the same way as the part of the confirmation of data transmission, acknowledgment will be sent from destination to source.

\subsection{Cluster Based Routing}

In this paper, a cluster based routing algorithm in which ant colony optimization is used for performing route optimization including route discovery and route maintenance is proposed. In this routing algorithm cluster heads are used to perform routing in order to provide effective routing. 
Cluster head is elected based on energy level and its distribution. This method is used in this paper since in this method, all nodes would be selected as a cluster head after $1 / \mathrm{p}$ round. Therefore, it helps efficient-energy saving of nodes since the nodes which has high remaining energy are elected as a cluster head. However, it does not consider unequal energy consumption of nodes by unequal clusters. The elected cluster head is not again selected as a cluster head during $1 / p$ rounds although the node has the most energy than others. It can be seen that the energy gap between a cluster head and a member node is higher during management of clustering. In this paper, cluster heads are elected not only based on energy level but also on the degree of mobility with equal probability. In this method, nodes with higher energy levels are given more importance in comparison with the nodes with less energy. The nodes which rarely move are preferred in comparison with the frequently moving nodes.

The agent based ants are used in this work for performing the proactive route discovery were the agents use ants to periodically gather the routing information between different source-destination pairs to reduce the route discovery latency. In this process, the various components involved are probabilistic routing that consists of probability computation and periodic route discovery, path grading and reactive route discovery. The major advantages of this routing scheme are the reduction of route discovery latency, avoiding the exchange of entire routing information, maintenance of multiple paths with different grades and reducing the movement of ants to the cluster-heads and cluster-gateways.

In this ant based routing scheme, every node maintains a pheromone table and a probability routing table. Pheromone values are maintained in all the nodes of the network for each destination-neighbor pairs. The pheromone values for all neighbors for a particular destination are initialized to the same value to make the search unbiased. These initial values of the pheromones change with respect to the number of ants moving towards a particular neighbor. The path in which more ants move towards a link is the optimal path to the destination. As more ants move, the particular pheromone entry increases and hence that neighbor gets more probability. The quality of the link life, energy depletion rate of neighbors and processing power of the neighbors affect the probability values of the neighbors.

The probability routing table for all destinations is maintained at nodes. If the node $\mathrm{n}$ has $\mathrm{k}$ neighbors, probability of selecting the neighbor $\mathrm{m}$ for the destination $\mathrm{d}$ is computed as in equation 1 .

$$
P_{d}=\frac{P_{m}}{\sum_{i=1}^{k} P_{i}}
$$

where $P_{d}$ is the probability of selecting the neighbor $m$ for destination $\mathrm{d}$ and $\mathrm{P}_{\mathrm{m}}$ is the individual probability of node $\mathrm{m}$. Probability in turn is computed as a function of pheromone and the link quality based on some heuristic.

The main advantage of cluster based routing is that it reduces the number of hops. Moreover, ants are used in the route discovery and route maintenance process. As a result, the performance metrics of applications that use this protocol achieve better quality of service in terms of end-to-end delay, packet delivery ratio and throughput.

\section{IMPLEMENTATION AND RESULTS}

The results obtained from this work are presented in three major sections namely pipelining, optimal routing with agents and cross layer design. In the first stage, the results obtained on pipelining show that with different number of data channels and different number of control channels it is possible to improve the throughput and reduce the delay and packet loss. In the second stage, results related to optimal routing are demonstrated to show the advantages of routing in wireless networks as a means to improve the overall throughput of the network. Results obtained from the un-clustered routing are also compared with the results of the optimized cluster based routing. In the third stage, results obtained with cross layer design are compared with the results obtained without cross layer design.

\subsection{Simulation Scenario}

In this work, the simulation models that are present in the NS2 library for the performance evaluation of networks have been used for validating the pipelining and clustering schemes. The NS2 library is a scalable simulation environment for wireless networks. In the simulation scenarios, the distributed coordination function (DCF) of IEEE 802.11 is used as the Medium Access Control (MAC) layer protocol first without pipelining and then with pipelining. The radio propagation range for each node is taken as $250 \mathrm{~m}$, and channel capacity as 4 Mbps. The propagation model used is the two-way propagation model. The random way point model has been used for node mobility and the nodes are placed in random positions.

AODV has been used as the routing protocol in all the evaluations because AODV is one of the most popular ondemand protocols. Analysis has been performed for low-density and high-density wireless networks in which the mobility varies from 1 meter per second to 10 meters per second. For low density, 128 nodes have been placed randomly in a roaming space of $1000 \mathrm{~m} * 1000 \mathrm{~m}$. For high density, 1024 nodes have been placed in a roaming space of $1000 \mathrm{~m} * 1000 \mathrm{~m}$. The Constant Bit Rate (CBR) sources use 512-byte data packets to generate the traffic. The number of source-destination pairs and the packet-sending rate in each pair is varied to change the offered load in the network. Each simulation was carried out for about 900 seconds. The results obtained were used to find the average values over 20 randomly generated node topologies with different seed values. The mobility speed follows the random way point movement and was set as $20 \mathrm{~cm} / \mathrm{second}$. The transport layer was taken as TCP for one set of applications and UDP was taken for other set of simulations performed for video transmission applications.

\subsection{Results Obtained From Pipelined Scheduling}

The results obtained from the simulation of two stage pipelining are shown in this section. The analysis has been carried out on the metrics throughput, delay and loss. Figure 3 shows the comparison of throughputs achieved using pipelining and without pipelining for different numbers of data channels. 


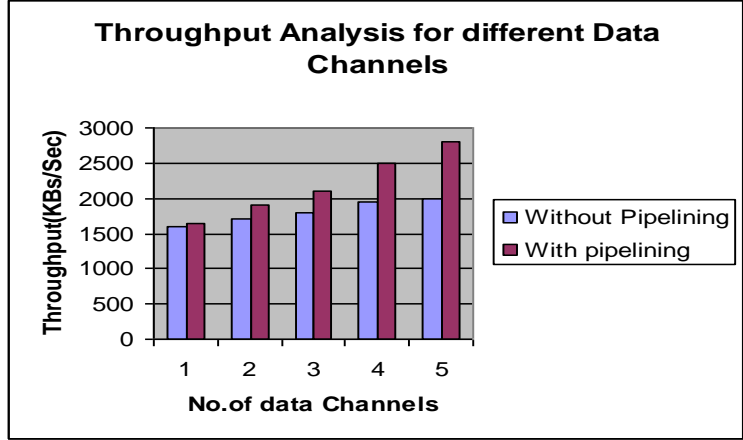

Fig 3: Throughput analysis for different number of data channels

From Figure 3, it can be observed that pipelining increases throughput of the system when it is supported with more number of data channels. Figure 4 shows the comparison of throughputs achieved using pipelining and without pipelining for different numbers of control channels.

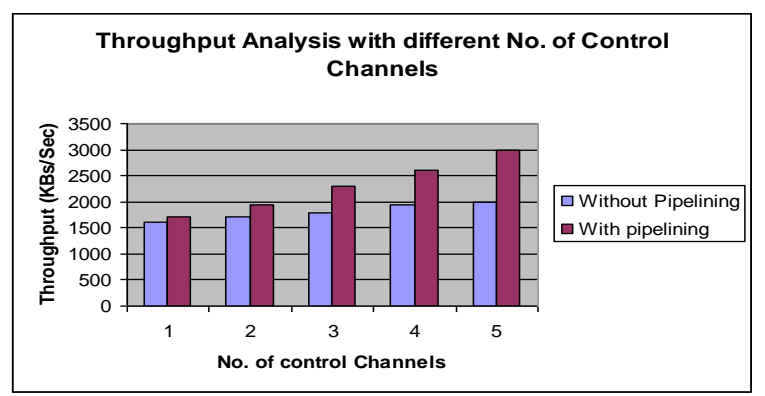

Fig 4: Throughput analyses for different number of control channels

From Figure 4, it can be observed that pipelining increases throughput of the system when it is supported with more number of control channels. Figure 5 shows the comparison of delays occurred when using pipelining and without pipelining for different numbers of data channels.

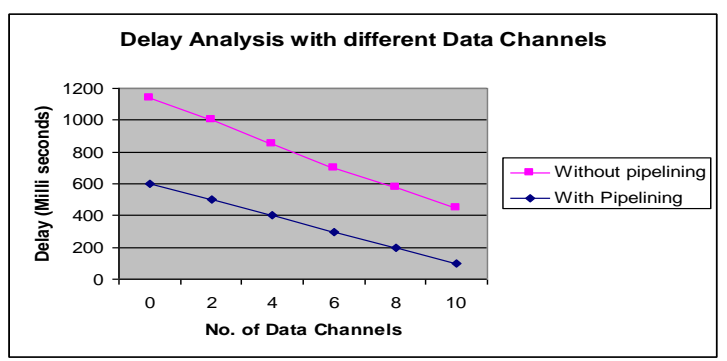

Fig 5: Delay analysis for different number of data channels

From Figure 5, it can be observed that pipelining decreases the delay of the system when it is supported with more number of data channels. Figure 6.4 shows the comparison of delays occurred when using pipelining and without pipelining for different numbers of control channels.

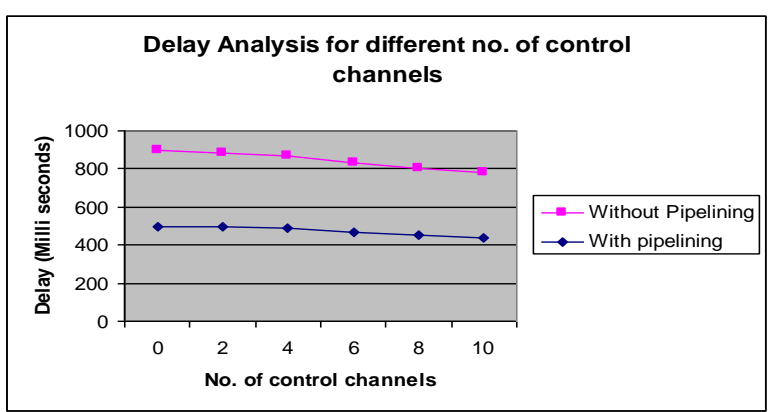

Fig 6: Delay analysis for different number of control channels

From Figure 6, it can be observed that pipelining decreases the delay in the system when it is supported with more number of control channels with pipelining. Figure 7 shows the packet loss analysis when pipelines are used and without pipelining.

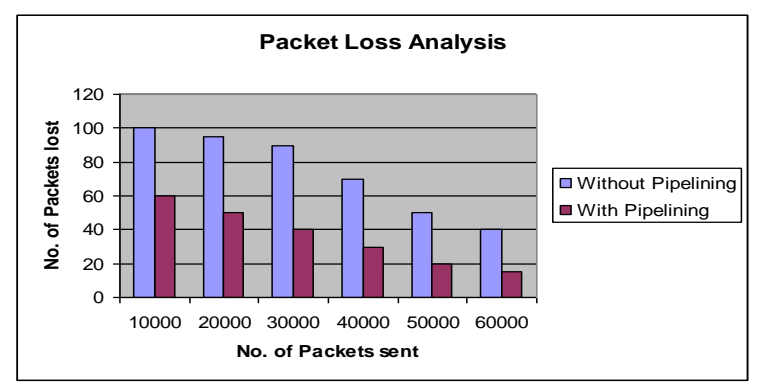

Fig 7: Packet Loss Analysis

From Figure 7, it can be observed that pipelining increases throughput of the system when it is supported with more number of data channels.

\subsection{Results From Cluster Based Routing}

The performance of this proposed cluster based routing algorithms have been analyzed based on performance of routing and overheads involved. Performance of routing and overheads are analyzed separately in high-density wireless networks and low-density wireless networks.

Performance analysis of the proposed cluster based routing scheme against the existing AODV has been performed based on packet delivery ratio, end-to-end delay and throughput. It has been observed that the applications running on the cluster based wireless network provide better performance than the applications running in flat wireless networks. This is due to the fact that in this proposed cluster based routing, route discovery and route maintenance are carried out only based on the cluster heads. Since route discovery and route maintenance are confined to the cluster-heads and cluster-gateways, the results such as reduction in average end-to-end delay, improvement in packet delivery ratio and throughput are achieved.

Figure 8 shows the comparison of packet delivery ratios between routing with AODV and optimized routing. 


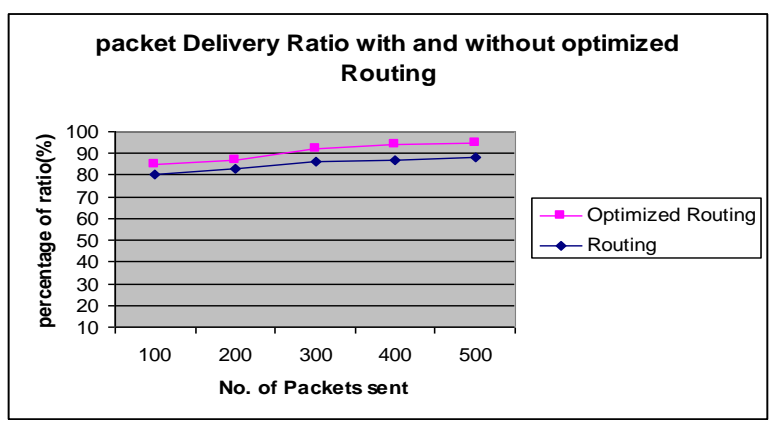

Fig 8: Packet delivery Ratio with Optimized Routing and Routing

From figure 8 , it can be seen that the cluster based optimal routing performs better than AODV in terms of packet delivery ratio.

\subsection{Ant Colony Optimization Based Clustering Algorithm}

In this work, an intelligent ant colony optimization has been proposed and implemented to reduce the clustering overhead and thereby to improve the network throughput when it is used for cross layer design of MAC layer and network layer. The performances of un-optimized and optimized clustering algorithms have been analyzed in terms of number of reaffiliations and load balancing factor in this thesis. From these experiments, it has been observed that the ant colony based optimization applied on the clustering algorithm has improved the performance of the wireless network considerably as shown in table 1 when compared against un-optimized clustering algorithm.

\subsection{Cross Layer Design of Wireless Networks}

The cross layer design scenario consists of tens of nodes including five servers and five client nodes that demands different services only after such services became available. The experiments have been carried out with varying number of nodes. The numbers of nodes considered are 10, 20, 30, 40 and 50. Figures 9 and 10 show that the performance of the proposed cross layer design approach is higher when compared with single layer design in terms of end-to-end delay and overhead respectively.

Figure 9 shows the comparison of packet delivery ratios of optimized routing and cross layer optimized routing.

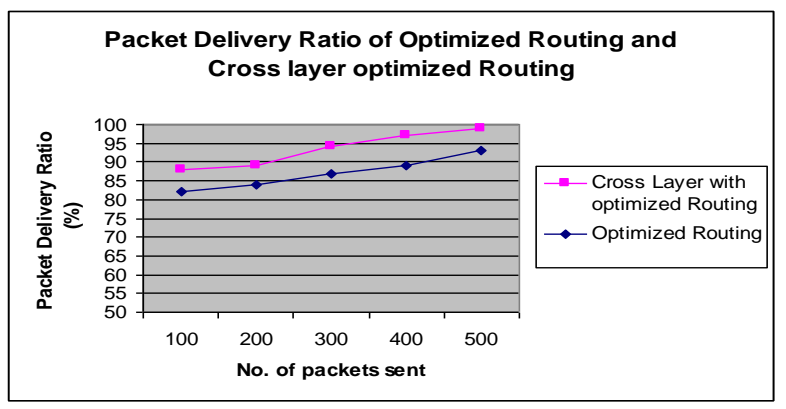

Fig 9: Packet delivery Ratio for Cross layer Design
From Figure 9, it can be observed that the packet delivery ratio is increased when cross layer design is followed.

Figure 10 shows the comparison of delay analysis for networks without cross layer design and network with cross layer design.

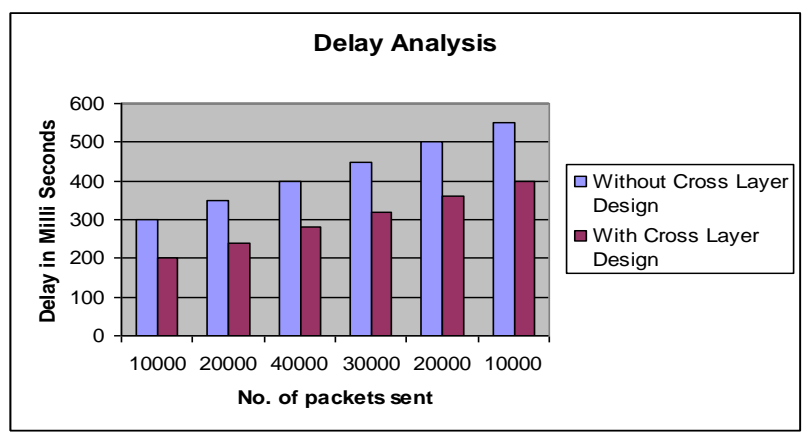

Fig 10: Delay Analysis for Cross layer design

From Figure 10, it can be observed that cross layer design reduces the delay over single layer design.

Table 1. End-to End Delay comparison for ant colony optimization

\begin{tabular}{|c|c|c|c|}
\hline $\begin{array}{c}\text { No. of } \\
\text { Nodes }\end{array}$ & $\begin{array}{c}\text { No. of } \\
\text { clusters }\end{array}$ & $\begin{array}{c}\text { No. of } \\
\text { reaffiliations } \\
\text { with AODV }\end{array}$ & $\begin{array}{c}\text { No. of } \\
\text { reaffiliations } \\
\text { with } \\
\text { Optimized } \\
\text { AODV }\end{array}$ \\
\hline 100 & 5 & 5 & 4 \\
\hline 200 & 10 & 10 & 8 \\
\hline 300 & 15 & 14 & 12 \\
\hline 400 & 20 & 18 & 16 \\
\hline 500 & 25 & 20 & 20 \\
\hline 600 & 30 & 26 & 21 \\
\hline 700 & 35 & 29 & 24 \\
\hline 800 & 40 & 35 & 26 \\
\hline 900 & 45 & 38 & \\
\hline
\end{tabular}

\section{CONCLUSIONS AND FUTURE WORKS}

In this paper, a cross layer network design approach has been proposed and implemented to improve the performance of wireless networks. For this purpose, new pipeline based MAC design principles have been proposed for effective scheduling and channel allocation. In addition, a cluster based ant colony optimization algorithm has been proposed for effective routing. This helps to improve the throughput and to reduce the delay. Moreover, experiments have been carried out by varying amount of network traffic. From the experiments conducted in this research work, it has been observed that the throughput of the system is increased by $5 \%$ when cross layer network design is employed. 
Future works in this direction could be the consideration of congestion control and flow control techniques for improving the network performance. In addition, fish school algorithm can be employed to perform route optimization in the network layer.

\section{REFERENCES}

[1] Sunil Kulkarni, Aravind Iyer and Catherine Rosenberg," An Address-Light, Integrated MAC and Routing Protocol for Wireless Sensor Networks ", IEEE/ACM Transactions on networking, Vol. 14, No. 4,pp.793-806, 2006.

[2] Jinhua Zhu, Xin Wang, and Dahai Xu, “A Unified MAC and Routing Framework for Multichannel Multi-interface Ad Hoc Networks", IEEE Transactions on Vehicular Technology, Vol. 59, No. 9, pp. 4589-4601, 2010.

[3] Michele Rossi and Michele Zorzi, "Integrated Cost-Based MAC and Routing Techniques for Hop Count Forwarding in Wireless Sensor Networks" IEEE Transactions on Mobile Computing, Vol. 6, No. 4, pp. 434-468, 2007.

[4] Chang, C., Tu, S.: Active route-maintenance protocol for signal-based communication path in ad hoc networks", Journal of Network and Computer Applications, 2002, pp.161-177.

[5] Kitae Nahm, Ahmed Helmy, C. C. Jay Kuo: Cross-Layer Interaction of TCP and Ad Hoc Routing Protocols in Multihop IEEE 802.11 Networks. IEEE Trans. Mob. Comput., Vol. 7, No.4, pp.458-469, 2008.

[6] Atef Abdrabou and Weihua Zhuang, "Statistial QoS routing for IEEE 802.11 multi-hop ad hoc networks", IEEE Transactions on Wireless Communications, Vol. 8, Issue No. 3, 2009 pp. 1542-1552.

[7] Correia, S.L.O.B. Celestino, J. Cherkaoui, O., "Mobiityaware Ant Colony Optimization Routing for Vehiular Ad hoc Networks", Wireless Communications and Networking Conference, 2009, pp.1125-1130.

[8] Shanti Chilukuri, Anirudha Sahoo, "DGRAM: A Delay Guaranteed Routing and MAC Protocol for Wireless Sensor Networks ”, Vol.9, No.10, 2009, pp.1407-1423.

[9] Laura Rosati, Matteo Berioli and Gianluca Reali, "On ant routing algorithms in ad hoc networks with critical connectivity", Ad-hoc Networks, Elsevier, 2008.

[10] Yung Yi, Gustavo de Veciana,, and Sanjay Shakkottai, "MAC Scheduling With Low Overheads by Learning Neighborhood Contention Patterns", IEEE/ACM Transactions On Networking, Vol. 18, No. 5, pp.16371650, 2010.

[11] Emad Shihab, Lin Cai, and Jianping Pan, "A Distributed Asynchronous Directional-to-Directional MAC Protocol for Wireless Ad Hoc Networks ", IEEE Transactions On Vehicular Technology, Vol. 58, No. 9, pp. 5124-5134, 2009.

[12] Elli Kartsakli, Nizar Zorba, Luis Alonso, and Christos V. Verikoukis," A Threshold-Selective Multiuser Downlink MAC Scheme for 802.11n Wireless Networks", IEEE Transactions on Wireless Communications, Vol. 10, No. 3, pp. 857-867, 2011.

[13] Liqi Shi, Abraham O. Fapojuwo, "TDMA Scheduling with Optimized Energy Efficiency and Minimum Delay in Clustered Wireless Sensor Networks", IEEE Transactions On Mobile Computing, Vol. 9, No. 7, pp.927-940, 2010.

[14] Hui Xu, Xianren Wu, Hamid R. Sadjadpour, J. J., "GarciaLuna-Aceves: A Unified Analysis of Routing Protocols in MANETs", IEEE Transactions on Communications, Vol. 58, No.3, pp.911-922, 2010. 